\title{
Recombinant truncated AniA of pathogenic Neisseria elicits a non-native immune response and functional blocking antibodies
}

\author{
Lucy K. Shewell ${ }^{1}$, Shan C. Ku², Benjamin L. Schulz ${ }^{2}$, Freda E.-C. Jen ${ }^{1}$, Tsitsi D. Mubaiwa ${ }^{1}$, \\ Margaret R. Ketterer ${ }^{3}$, Michael A. Apicella ${ }^{3}$, and Michael P. Jennings $\mathbf{s}^{*}, 1$ \\ ${ }^{1}$ Institute for Glycomics, Griffith University, Gold Coast, Queensland 4222, Australia \\ ${ }^{2}$ School of Chemistry and Molecular Biosciences, University of Queensland, St Lucia, Brisbane, \\ Queensland 4072, Australia
}

${ }^{3}$ Department of Microbiology, University of lowa, 51 Newton Rd., BSB 3-403, lowa City, IA 52242 , USA

\section{Abstract}

AniA of the pathogenic Neisseria is glycosylated in its C-terminal repeat region by the pilin glycosylation $(\mathrm{pgl})$ pathway. AniA appears to be unique among bacterial nitrite reductases as it contains an $\mathrm{N}$-terminal extension that includes a lipid modification site as well as a $\mathrm{C}$-terminal extension that is glycosylated. Immunising with various glycoforms of the AniA protein demonstrated a strong humoral immune response to the basal monosaccharide. In addition, when animals were immunised with a truncated form of AniA, completely lacking the glycosylated Cterminal region, the antibody response was directed against AniA regardless of the glycosylation state of the protein. Immuno-SEM confirmed that AniA is expressed on the cell surface in $N$. gonorrhoeae. Antisera generated against a truncated, non-glycosylated, recombinant form of the AniA protein are capable of blocking nitrite reductase function in a whole cell assay. We propose that recombinant modified AniA has potential as a vaccine antigen for $N$. gonorrhoeae.

\section{Keywords}

glycosylation; AniA; nitrite reductase; Neisseria meningitidis; Neisseria gonorrhoeae

\section{INTRODUCTION}

The pathogenic Neisseria species consist of N. meningitidis, which causes bacterial meningitis and sepsis, and $N$. gonorrhoeae, the causative agent of the sexually transmitted disease gonorrhoea. Currently there is no vaccine to prevent infection by $N$. gonorrhoeae, and there are now reports of $N$. gonorrhoeae strains that are resistant to the last remaining first-line treatment option for gonorrhea, the third-generation cephalosporin ceftriaxone [1], highlighting the pressing need for the development of gonoccocal vaccines.

*Correspondence to: Michael Jennings, Institute for Glycomics, Griffith University, Gold Coast Campus, QLD4222, Australia, Ph: +61 75552 7050, Fax: +61 75552 8098, m.jennings@ griffith.edu.au. 
In recent studies, we have identified an additional outer membrane glycoprotein in pathogenic Neisseria, the copper-containing nitrite reductase AniA, formerly known as Pan $1[2 ; 3]$, that is glycosylated in its C-terminal region by the $p g l$ pathway [4]. Both $N$. gonorrhoeae and $N$. meningitidis contain the internal enzymatic steps of the denitrification pathway in which nitrite is reduced to nitric oxide (NO) by AniA [2], and nitric oxide is reduced to nitrous oxide $\left(\mathrm{N}_{2} \mathrm{O}\right)$ by the nitric oxide reductase, NorB [5]. AniA is the major anaerobically induced outer membrane lipoprotein in $N$. gonorrhoeae $[3 ; 6]$ and is essential for the growth and survival of $N$. gonorrhoeae under oxygen-limiting conditions [2]. It has also been demonstrated that a strong immune response is generated against AniA in patients with various gonococcal infections [7]. N. gonorrhoeae has been shown to form biofilms in vitro and in vivo during cervical infection which may be associated with persistent gonococcal infection in asymptomatic women [8]. Expression of AniA is highly upregulated during biofilm growth of the gonococcus $[9 ; 10]$ and anaerobic respiration mediated by AniA is essential to normal biofilm formation and is widespread in the substratum of gonococcal biofilms. The cellular localisation of AniA is controversial with one model suggesting it is directed towards the periplasm [11], while a second model proposes that AniA is directed to the outside of the cell [12].

In this study we investigate the localisation of the AniA protein and examine the impact of glycosylation on the immune response to AniA as an adjunct to assessing its vaccine potential.

\section{MATERIALS AND METHODS}

\section{Bacterial strains, plasmids and growth conditions}

All bacterial strains and conditions used in this study are listed in Supplementary Table 1 and associated text in Supplementary Experimental Procedures.

\section{Construction and purification of glycoforms of FLAG-tagged AniA from N. meningitidis C311 strains}

Strains expressing FLAG-tagged glycoforms of AniA were constructed as described in [4] with details provided in Supplementary Experimental Procedures.

\section{Production of polyclonal antisera against glycoforms of AniA}

Groups of 5 BALB/c mice were immunised on days 0,21 and 28 with $5 \mu \mathrm{g}$ of either FLAGtagged AniA glycosylated with the DATDH monosaccharide, non-glycoslyated FLAGtagged AniA or truncated FLAG-tagged AniA lacking the C-terminal glycosylation region. Following this immunisation schedule, terminal bleeds were collected and the serum from each mouse was harvested. These antisera were analysed by western blotting and ELISA as described in Supplementary Experimental Procedures.

\section{Expression and purification of His-tagged truncated AniA proteins}

DNA sequences encoding AniA with various truncations of the $\mathrm{N}$ - and $\mathrm{C}$-terminal regions (Antigens $1-8$ ) were amplified from $N$. gonorrhoeae 1291 genomic DNA using the primers described in Supplementary Table 2. Primers were designed based on the aniA sequence 
from the N. gonorrhoeae 1291 genome sequence (Broad Institute NGAG_01981). Digested PCR products were cloned into pET-15b (Novagen) to create the expression plasmid constructs described in Supplementary Table 1. E. coli BL21 (DE3) cells were transformed with the plasmid constructs and the His-tagged proteins were expressed and purified using TALON Metal Affinity Resin (Clontech) according to the manufacturer's instructions.

\section{Production of polyclonal antisera against recombinant His-tagged AniA proteins}

8 New Zealand white rabbits were immunised on days 0,21, 42 and 63 with 100 $\mu \mathrm{g}$ of each of the purified His-tagged truncated AniA proteins (Antigens $1-8$ ). Following this immunisation schedule, terminal bleeds were collected and the serum from each rabbit was harvested.

\section{Isolation of outer membrane proteins}

Outer membrane proteins were isolated essentially as described by [13].

\section{Immuno-Scanning Electron Microscopy (SEM) analysis}

N. gonorrhoeae 1291 cells used for immuno-SEM were grown anaerobically with $2 \mathrm{mM}$ $\mathrm{NaNO}_{2}$. Samples were incubated with pre-immune rabbit serum (1: 1000 dilution) or antiAniA polyclonal rabbit serum (raised against recombinant His-tagged AniA - Antigen 5) (1: 1000 dilution) followed by incubation with anti-rabbit gold conjugated antibody. The images were collected by scanning EM on the Hitachi S4800.

\section{Trypsin digestion of surface exposed proteins}

Surface exposed proteins of $N$. gonorrhoeae 1291 were digested according to RodriguezOrtega et al [14], with modifications as described in Supplementary Experimental procedures.

\section{Nitrite utilisation assays}

Antisera raised against recombinant His-tagged AniA proteins (Antigens 1 - 8) were used in nitrite utilisation assays as described in Supplementary Experimental Procedures.

\section{RESULTS}

\section{Investigation of the immunogenicity of the AniA glycoforms in N. meningitidis C311}

Typically bacterial nitrite reductases are periplasmic soluble enzymes [15]. The core region of the AniA nitrite reductase from N. meningitidis $\mathrm{C} 311$ is homologous to other characterised bacterial nitrite reductases, containing a number of conserved residues essential to the function of the enzyme, while the $\mathrm{N}$ - and C-termini of this protein appear to be distinct (Supplementary Fig. 1). The N-terminal region of AniA from N. meningitidis and N. gonorrhoeae contains a lipoprotein signal peptidase II-processing site (ALAAC) and regions of homology to two other Neisseria outer membrane lipoproteins, Lip/H.8 and Laz [3] (Supplementary Fig. 2A - underlined). This suggests that AniA from these Neisseria species, similar to Lip/H.8 and Laz, is an outer membrane protein anchored via this lipid modified $\mathrm{N}$-terminus. AniA has been identified as a glycoprotein and the $\mathrm{C}$-terminus 
encompasses the glycosylated serine residues which are present within the sequence AASAP [4] (Supplementary Fig. 2A).

To investigate the role of the glycosylation of AniA in the immune response to this protein, mice were immunised with AniA proteins purified from different $\mathrm{pgl}$ mutant strains and the reactivity of the resulting antisera was analysed against outer membrane proteins isolated from the wild-type strain, as well as selected $p g l$ mutants (Fig. 1A). Mice were immunised with AniA purified from C311 pglA, which was glycosylated with a truncated glycan consisting of the 2,4-diacetimido-2,4,6-trideoxyhexose (DATDH) monosaccharide, with non-glycosylated AniA purified from C311 pglL, as this mutant is missing the PglL oligosaccharyltransferase, and with a truncated form of AniA completely lacking the Cterminal glycosylated region. Antisera raised against AniA glycosylated with the DATDH monosaccharide were the most reactive with AniA from the pglA mutant strain (Fig. 1B). Antisera generated against non-glycosylated AniA (Fig. 1C) and against the truncated form of AniA, lacking the C-terminal glycosylation region (Fig. 1D), were found to react equally with all glycoforms of AniA.

The reactivity of antisera from individual mice immunised with the different glycoforms of AniA was assessed in an ELISA against a recombinant, truncated form of AniA expressed in E. coli, lacking both the N-terminus and the glycosylated C-terminus (See Supplementary Fig. 2B - Antigen 5). Mice immunised with the truncated form of AniA were found to have the highest mean titre against the truncated AniA protein whereas those mice immunised with AniA glycosylated with the monosaccharide were found to have the lowest mean titre (Fig. 1F). The difference between the mean titres of these two groups was statistically significant $(p$ value $=0.0433)$.

\section{AniA is expressed on the cell surface of $\boldsymbol{N}$. gonorrhoeae}

It has been shown that AniA is required for the growth and survival of $N$. gonorrhoeae under oxygen-limiting conditions [2] and is essential for optimal biofilm formation in this species [9]. Furthermore, antibodies against AniA have been detected in patients with various gonococcal infections [7]. While $34 \%$ of $N$. meningitidis clinical isolates contain a frame-shift mutation within the AniA coding sequence resulting in loss of expression, 100\% of $N$. gonorrhoeae strains examined were predicted to express full length AniA [4;16]. These findings taken together suggest that AniA of $N$. gonorrhoeae is expressed in vivo and may be important to the survival of the gonococcus in the human host. Therefore, we focused on AniA of $N$. gonorrhoeae for the remainder of this study and investigated the potential of AniA as a vaccine antigen for N. gonorrhoeae.

To confirm that AniA is on the surface of the cell immuno-SEM was conducted and showed surface labelling with anti-AniA polyclonal antiserum of $N$. gonorrhoeae 1291 cells grown anaerobically indicating that AniA is surface exposed (Fig. 2B). In further studies whole, intact $N$. gonorrhoeae 1291 cells grown anaerobically were treated with the protease tryspin. AniA was found to be susceptible to digestion with trypsin (Fig. 2C) verifying that AniA is exposed on the cell surface in $N$. gonorrhoeae. Treatment of cells with trypsin did not affect cell viability, as assessed by the determination of CFUs/ml before and after trypsin digestion (Supplementary Table. 3). 


\section{Polyclonal antisera raised against truncated, recombinant AniA is capable of functional blocking}

Based on our findings that the removal of the AniA glycan generated an antibody response that was independent of the glycoyslation state of the protein, we created a series of recombinant AniA proteins with various truncations of the $\mathrm{N}$ - and $\mathrm{C}$-terminal regions in an E. coli host (Supplementary Fig. 2B) to determine the minimum antigen required to elicit a functional blocking humoral immune response, directed at regions that are essential to the function of this enzyme, such as the type I and type II copper centres, or by inhibiting electron donation.

The ability of these antisera to block AniA nitrite reductase acitivity in N. gonorrhoeae was assessed in nitrite utilisation assays. Antiserum raised against Antigen 1 showed inhibition of AniA activity (Fig. 3). Antisera raised against the remaining antigens did not show inhibition of AniA activity (data not shown). These data suggest that by removing the Cterminal region of AniA, antibodies can be generated that are directed against the core, catalytic domain of the protein that are capable of inhibiting nitrite reductase activity.

\section{DISCUSSION}

In contrast to most other characterised bacterial nitrite reductases, which are soluble periplasmic enzymes, the copper-containing nitrite reductase from the pathogenic Neisseria species, AniA, contains a lipid-modified $\mathrm{N}$-terminal region, which is homologous to the meningococcal and gonococcal outer membrane proteins Lip/H.8 and Laz [3]. It has been shown that a proportion of $N$. meningitidis strains have lost expression of AniA due to a frame-shift mutation resulting in premature termination of the protein [4] and that various amino acid point mutations, insertions and deletions are present in the AniA sequences from a range of clinical isolates, some of which may affect the catalytic regions and thus the function of the protein [16]. N. meningitidis is dependent on the presence of oxygen for growth and cannot grow under strictly anaerobic conditions [17]. Although some strains may use denitrification of nitrite to nitric oxide to supplement a limited supply of oxygen as a respiratory electron acceptor [17], it appears that AniA nitrite reductase activity is not essential to the survival or pathogenesis of $N$. meningitidis. Thus it has been proposed that $N$. meningtidis is evolving into a species that no longer requires the reduction of nitrite by AniA to supplement growth and is better suited to a more aerobic environment [18]. This is in contrast to the situation in $N$. gonorrhoeae where AniA seems to be essential. Expression of aniA is maintained in all strains surveyed thus far $[4 ; 19]$. The differences in the requirement for AniA in the meningococcus and the gonococcus may be due to the distinct niches colonised by these two human pathogens [18]. N. meningitidis colonises the more oxygen rich epithelium of the upper respiratory tract while $N$. gonorrhoeae colonies the mucosal epithelium of the human urogenital tract, which is presumed to be an oxygenlimited environment. A number of findings suggest that $N$. gonorrhoeae encounters anaerobic conditions during colonisation including the frequent isolation of gonococci with facultative and obligate anaerobes [20] and the strong antibody response generated against AniA in patients with various gonococcal infections [7]. AniA expression is tightly regulated by oxygen availability and is virtually undetectable in gonococcal cells grown 
aerobically [21]. Therefore it appears that the ability to adapt to and grow in an oxygendepleted environment is an important aspect of $N$. gonorrhoeae colonisation, and this ability is dependent on AniA nitrite reductase activity [2]. For these reasons we focused on AniA of N. gonorrhoeae in this study.

Two models have been proposed for the localisation of AniA: one arguing that AniA is directed towards the periplasm [11], while the second suggests that AniA is directed to the outside of the cell [12]. We have demonstrated by immuno-SEM and trypsin digestion of whole, viable cells that AniA is on the cell surface in N. gonorrhoeae. However, the possibility remains that there is a population of AniA that is directed towards the periplasm as trypsin digestion of surface exposed proteins failed to completely digest all of the AniA protein. This may indicate that a proportion of AniA is directed towards the periplasm and is protected from trypsin cleavage. Alternatively, particular residues of AniA may be inaccessibile to digestion with trypsin due to higher order protein structure. This is commonly observed for proteins in their native state [22] and it has been shown that the soluble domain of AniA from pathogenic Neisseria exists as a homotrimer [23]. The fact that we were able to inhibit a significant proportion of AniA nitrite reductase activity with Anti-AniA antiserum suggests that if a population of AniA is directed towards the periplasm it may not be functional.

Another unique feature of the AniA nitrite reductase from Neisseria is the glycosylated Cterminus. Interestingly, we found that the antibody response was directed primarily towards the basal monosaccharide of the AniA glycoprotein when it is presented to the immune system, with minimal antibodies being produced against the remainder of the protein, suggesting that this glycan is immunodominant. These findings are consistent with our previous study [24], as well as that of others [25], demonstrating an immune response to the Neisseria $O$-linked glycan. This antibody response was found to be glycan-specific with antisera raised against AniA glycosylated with the DATDH monosaccharide reacting strongly with AniA glycosylated with the DATDH monosaccharide and only weakly with AniA glycosylated with the trisaccharide molecule. These findings support the hypothesis that a function of AniA glycosylation may be to provide an immune evasion mechanism whereby the glycan acts as an immunological 'decoy' to reduce the relative antibody response generated against the functional polypeptide region of AniA. The pathogenic Neisseria species have the ability to display a variety of $O$-linked glycans due to the presence or absence of $p g l$ genes encoding glycosyltransferases including $p g l B, p g l C$ and $p g l D$ and due to the phase-variable expression of $p g l A, p g l E, p g l I$ and $p g l H$. This can result in strains that are capable of expressing at least 13 different glycoforms $[24 ; 26 ; 27 ; 28]$. Switching between the expression of various glycoforms of AniA may provide the bacteria with a mechanism to escape glycan specific antibodies developed by the host.

Moreover, we showed that by removing the glycan of AniA, as well as the entire C-terminal glycosylated region, a non-native immune response was elicited against the core region of the protein regardless of the glycosylation state. The flexible, C-terminal glycosylated region of AniA may serve to 'shield' the functional region of the protein from immune exposure and by removing the glycan that typically 'masks' the conserved epitopes of AniA an immune response can be generated against the functional protein core that would not 
normally occur. We were able to demonstrate this further by producing functional blocking antibodies directed against the core region of the AniA protein by immunising animals with a recombinant form of AniA completely lacking the glycosylated C-terminus.

In summary, we find that AniA is surface exposed, that the monosaccharide of the AniA glycan is immunodominant and that removal of this glycan generates a non-native immune response against the core of the protein that produces antibodies capable of functional blocking. As AniA is essential for the growth and survival of $N$. gonorrhoeae under oxygenlimited conditions [2] and for biofilm formation [29], we therefore propose that modified AniA has potential as a vaccine antigen for $N$. gonorrhoeae and warrants further investigation.

\section{Supplementary Material}

Refer to Web version on PubMed Central for supplementary material.

\section{Acknowledgments}

This work was supported by NHMRC Program Grant 565526 and by a Helen C. Levitt Endowed Annual Visiting Professorship to M.P.J., NHMRC CDF APP1031542 to B.L.S and by NIAID Grant AI045728 to M.A.A. We thank Virginia Clark for providing anti-AniA mAb.

\section{References}

1. Ohnishi M, Golparian D, Shimuta K, Saika T, Hoshina S, Iwasaku K, Nakayama S, Kitawaki J, Unemo M. Is Neisseria gonorrhoeae initiating a future era of untreatable gonorrhea?: detailed characterization of the first strain with high-level resistance to ceftriaxone. Antimicrob Agents Chemother. 55:3538-45. [PubMed: 21576437]

2. Mellies J, Jose J, Meyer TF. The Neisseria gonorrhoeae gene aniA encodes an inducible nitrite reductase. Mol Gen Genet. 1997; 256:525-32. [PubMed: 9413436]

3. Hoehn GT, Clark VL. The major anaerobically induced outer membrane protein of Neisseria gonorrhoeae, Pan 1, is a lipoprotein. Infect Immun. 1992; 60:4704-8. [PubMed: 1398981]

4. Ku SC, Schulz BL, Power PM, Jennings MP. The pilin O-glycosylation pathway of pathogenic Neisseria is a general system that glycosylates AniA, an outer membrane nitrite reductase. Biochem Biophys Res Commun. 2009; 378:84-9. [PubMed: 19013435]

5. Householder TC, Fozo EM, Cardinale JA, Clark VL. Gonococcal nitric oxide reductase is encoded by a single gene, nor $B$, which is required for anaerobic growth and is induced by nitric oxide. Infect Immun. 2000; 68:5241-6. [PubMed: 10948150]

6. Clark VL, Campbell LA, Palermo DA, Evans TM, Klimpel KW. Induction and repression of outer membrane proteins by anaerobic growth of Neisseria gonorrhoeae. Infect Immun. 1987; 55:135964. [PubMed: 3106220]

7. Clark VL, Knapp JS, Thompson S, Klimpel KW. Presence of antibodies to the major anaerobically induced gonococcal outer membrane protein in sera from patients with gonococcal infections. Microb Pathog. 1988; 5:381-90. [PubMed: 3148817]

8. Steichen CT, Shao JQ, Ketterer MR, Apicella MA. Gonococcal cervicitis: a role for biofilm in pathogenesis. J Infect Dis. 2008; 198:1856-61. [PubMed: 18973432]

9. Falsetta ML, Bair TB, Ku SC, Vanden Hoven RN, Steichen CT, McEwan AG, Jennings MP, Apicella MA. Transcriptional profiling identifies the metabolic phenotype of gonococcal biofilms. Infect Immun. 2009; 77:3522-32. [PubMed: 19528210]

10. Phillips NJ, Steichen CT, Schilling B, Post DM, Niles RK, Bair TB, Falsetta ML, Apicella MA, Gibson BW. Proteomic analysis of Neisseria gonorrhoeae biofilms shows shift to anaerobic 
respiration and changes in nutrient transport and outermembrane proteins. PLoS One. 2012; 7:e38303. [PubMed: 22701624]

11. Deeudom M, Koomey M, Moir JW. Roles of c-type cytochromes in respiration in Neisseria meningitidis. Microbiology. 2008; 154:2857-64. [PubMed: 18757819]

12. Cardinale JA, Clark VL. Expression of AniA, the major anaerobically induced outer membrane protein of Neisseria gonorrhoeae, provides protection against killing by normal human sera. Infect Immun. 2000; 68:4368-9. [PubMed: 10858263]

13. van der Ley P, Heckels JE, Virji M, Hoogerhout P, Poolman JT. Topology of outer membrane porins in pathogenic Neisseria spp. Infect Immun. 1991; 59:2963-71. [PubMed: 1652557]

14. Rodriguez-Ortega MJ, Norais N, Bensi G, Liberatori S, Capo S, Mora M, Scarselli M, Doro F, Ferrari G, Garaguso I, Maggi T, Neumann A, Covre A, Telford JL, Grandi G. Characterization and identification of vaccine candidate proteins through analysis of the group A Streptococcus surface proteome. Nat Biotechnol. 2006; 24:191-7. [PubMed: 16415855]

15. Rinaldo, S.; Cutruzzola, F. Nitrite reductases in dentrification. In: Bothe, H.; Ferguson, S.; Newton, WE., editors. Biology of the Nitrogen Cycle. Elsevier; Amsterdam: 2007. p. 37-55.

16. Stefanelli P, Colotti G, Neri A, Salucci ML, Miccoli R, Di Leandro L, Ippoliti R. Molecular characterization of nitrite reductase gene (aniA) and gene product in Neisseria meningitidis isolates: is aniA essential for meningococcal survival? IUBMB Life. 2008; 60:629-36. [PubMed: 18618739]

17. Rock JD, Mahnane MR, Anjum MF, Shaw JG, Read RC, Moir JW. The pathogen Neisseria meningitidis requires oxygen, but supplements growth by denitrification. Nitrite, nitric oxide and oxygen control respiratory flux at genetic and metabolic levels. Mol Microbiol. 2005; 58:800-9. [PubMed: 16238628]

18. Moir JW. A snapshot of a pathogenic bacterium mid-evolution: Neisseria meningitidis is becoming a nitric oxide-tolerant aerobe. Biochem Soc Trans. 2011; 39:1890-4. [PubMed: 22103546]

19. Barth KR, Isabella VM, Clark VL. Biochemical and genomic analysis of the denitrification pathway within the genus Neisseria. Microbiology. 2009; 155:4093-103. [PubMed: 19762442]

20. Burnakis TG, Hildebrandt NB. Pelvic inflammatory disease: a review with emphasis on antimicrobial therapy. Rev Infect Dis. 1986; 8:86-116. [PubMed: 2937130]

21. Householder TC, Belli WA, Lissenden S, Cole JA, Clark VL. cis- and trans-acting elements involved in regulation of aniA, the gene encoding the major anaerobically induced outer membrane protein in Neisseria gonorrhoeae. J Bacteriol. 1999; 181:541-51. [PubMed: 9882668]

22. Hubbard SJ. The structural aspects of limited proteolysis of native proteins. Biochim Biophys Acta. 1998; 1382:191-206. [PubMed: 9540791]

23. Boulanger MJ, Murphy ME. Crystal structure of the soluble domain of the major anaerobically induced outer membrane protein (AniA) from pathogenic Neisseria: a new class of coppercontaining nitrite reductases. J Mol Biol. 2002; 315:1111-27. [PubMed: 11827480]

24. Warren MJ, Roddam LF, Power PM, Terry TD, Jennings MP. Analysis of the role of pglI in pilin glycosylation of Neisseria meningitidis. FEMS Immunol Med Microbiol. 2004; 41:43-50. [PubMed: 15094166]

25. Borud B, Aas FE, Vik A, Winther-Larsen HC, Egge-Jacobsen W, Koomey M. Genetic, structural, and antigenic analyses of glycan diversity in the O-linked protein glycosylation systems of human Neisseria species. J Bacteriol. 2010; 192:2816-29. [PubMed: 20363948]

26. Power PM, Roddam LF, Rutter K, Fitzpatrick SZ, Srikhanta YN, Jennings MP. Genetic characterization of pilin glycosylation and phase variation in Neisseria meningitidis. Mol Microbiol. 2003; 49:833-47. [PubMed: 12864863]

27. Jennings MP, Virji M, Evans D, Foster V, Srikhanta YN, Steeghs L, van der Ley P, Moxon ER. Identification of a novel gene involved in pilin glycosylation in Neisseria meningitidis. Mol Microbiol. 1998; 29:975-84. [PubMed: 9767566]

28. Borud B, Viburiene R, Hartley MD, Paulsen BS, Egge-Jacobsen W, Imperiali B, Koomey M. Genetic and molecular analyses reveal an evolutionary trajectory for glycan synthesis in a bacterial protein glycosylation system. Proc Natl Acad Sci U S A. 2011; 108:9643-8. [PubMed: 21606362] 
29. Falsetta ML, McEwan AG, Jennings MP, Apicella MA. Anaerobic metabolism occurs in the substratum of gonococcal biofilms and may be sustained in part by nitric oxide. Infect Immun. 2010; 78:2320-8. [PubMed: 20231417] 

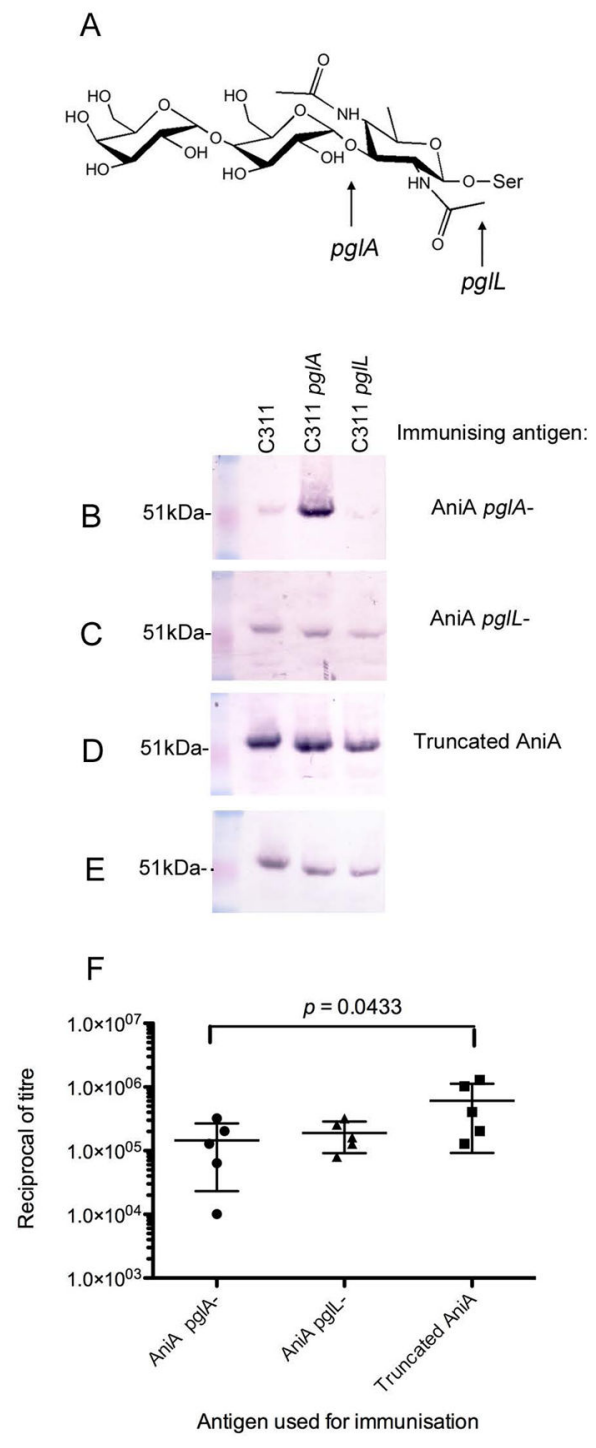

Figure 1. Analysis of the immunogenicity of the glycoforms of AniA

(A) Structure of the $N$. meninigitidis $O$-linked trisaccharide molecule Gal ( $\beta 1-4)$ Gal (a1-3) 2,4-diacetamido-2,4,6-trideoxyhexose (DATDH). The arrows indicate the truncations of the trisaccharide structure in $p g l A$ and $p g l L$ mutant strains. (B) A typical result from western blot analyses of antisera from mice immunised with AniA glycosylated with the DATDH monosaccharide against outer membrane proteins from C311 (AniA glycosylated with a trisaccharide molecule), C311 pglA (AniA glycosylated with a monosaccharide) and C311 pglL (non-glycosylated AniA). AniA is detected at approximately $51 \mathrm{kDa}$ in C311 and at slightly lower molecular weights in C311 pglA and in C311 pglL due to glycosylation with the monosaccharide and loss of the glycan, respectively. (C) A typical result from western blot analyses of antisera from mice immunised with non-glycosylated AniA against outer membrane proteins from C311, C311 pglA and C311 pglL. (D) A typical result from western blot analyses of antisera from mice immunised with truncated AniA lacking the C-terminal glycosylation region (1: 100) against outer membrane proteins from C311, C311 pglA and 
C311 pglL. (E) Western blot analysis of outer membrane proteins from C311, C311 pglA and C311 pglL using an anti-AniA mAb to show that equal amounts of the AniA protein were present in each sample. (F). Immunisation with AniA lacking the C-terminus elicits a greater antibody response against the non-glycan epitopes of the protein. The reciprocal of the antibody titres from mice immunised with AniA glycosylated with the monosaccharide (AniA pglA-), non-glycosylated AniA (AniA pglL-) and AniA lacking the C-terminus (Truncated AniA) against recombinant truncated AniA (Antigen 5) are shown. Error bars represent \pm 1 standard deviation from the mean. A statistically significant difference was detected between the mean antibody titre of the group immunized with AniA pglA- and the group immunised with Truncated AniA $(p=0.0433)$. 

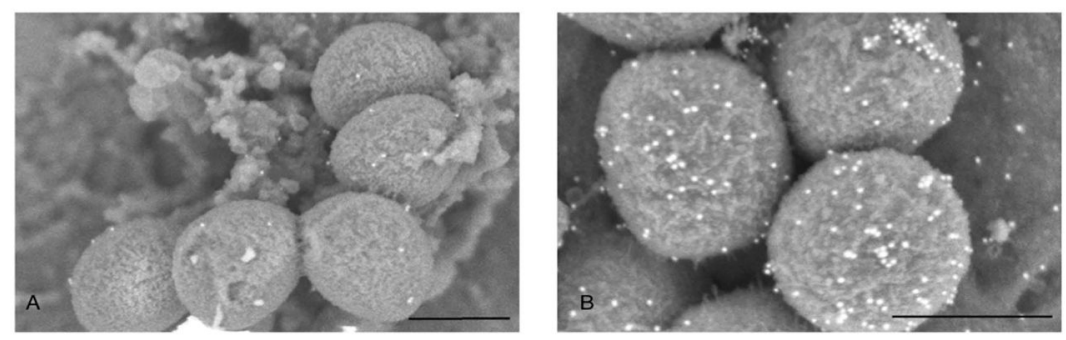

C

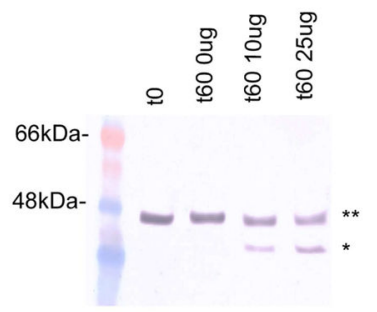

Figure 2. AniA is expressed on the cell surface in N. gonorrhoeae 1291

Examination of $N$. gonorrhoeae 1291 cells grown anaerobically immunolabelled with (A) pre-immune rabbit serum or (B) anti-AniA polyclonal rabbit serum using SEM. Scale bars indicate 500nm. (C). Western blot analysis of whole, intact anaerobicaly grown $N$. gonorrhoeae 1291 cells treated with increasing concentrations of trypsin using antiAniA polyclonal rabbit serum. $* *=$ Full-length AniA, $*=$ Digested AniA. No significant differences between the CFUs/ml at t0 and at $60 \mathrm{mins}$ for each of the samples were detected (Supplementary Table 3). 


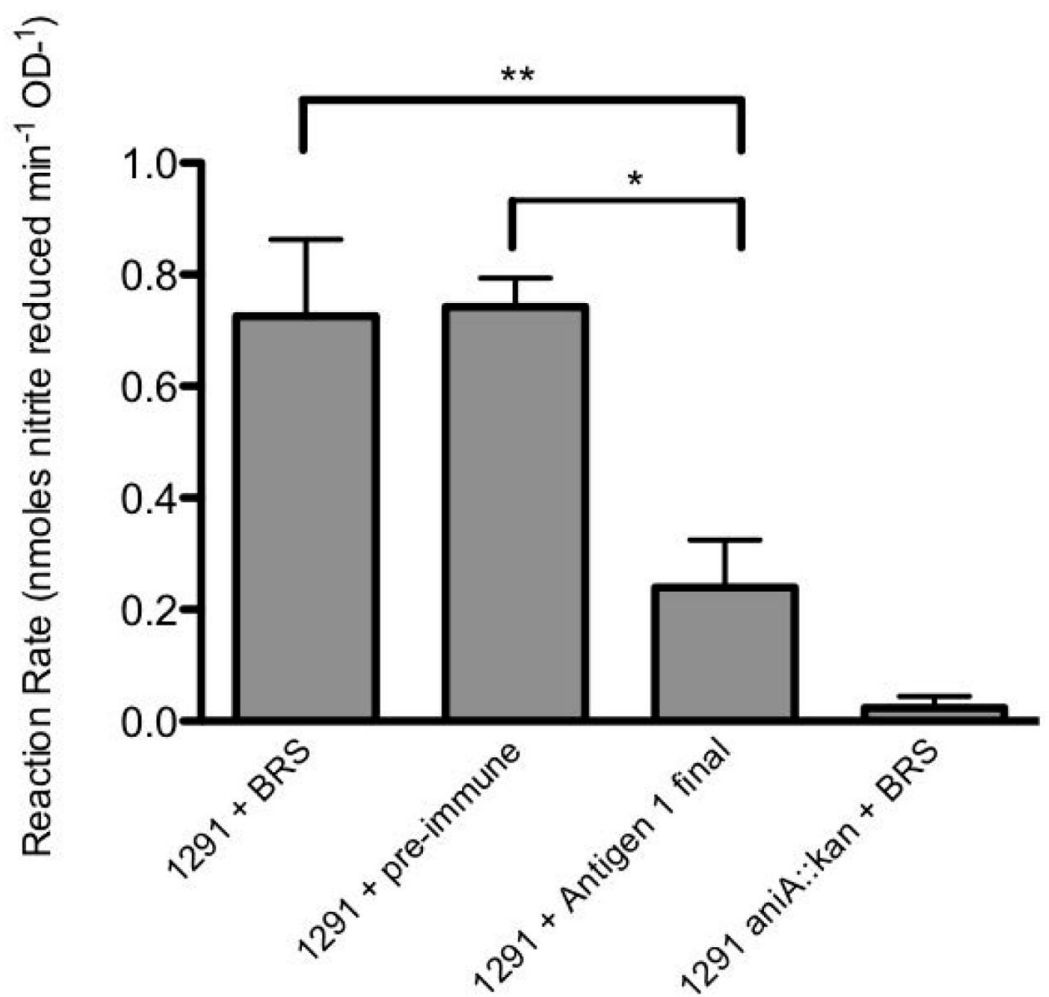

Figure 3. Polyclonal rabbit serum raised against Antigen 1 is able to block AniA nitrite reductase activity of $N$. gonorrhoeae 1291 cells

Bars represent AniA activity expressed as the mean reaction rate (nmoles of nitrite reduced $/ \mathrm{min} / \mathrm{OD}_{600}$ ) from triplicate samples over the course of a nitrite utilisation assay. Samples include 1291 cells pre-incubated with 1:10 baby rabbit serum (BRS) (1291 + BRS), 1291 cells pre-incubated with 1:10 pre-immune rabbit serum $(1291+$ pre-immune $), 1291$ cells pre-incubated with 1:10 polyclonal rabbit serum raised against Antigen $1(1291+$ Antigen 1 final) and 1291 aniA::kan cells pre-incubated with 1: 10 BRS (1291 aniA::kan + BRS). Error bars represent +1 standard deviation from the mean. The reaction rate for cells pre-incubated with polyclonal rabbit serum raised against Antigen 1 was significantly lower compared to cells pre-incubated with either BRS $(* * p=0.0443)$ or with pre-immune rabbit serum $(* p=0.0144)$. 